\title{
Detection of Second Canals in Mesial Root of Maxillary First Molars Using Different Evaluation Methods (In Vitro Study)
}

\author{
Amer A. Mohammed \\ B.D.S., M.Sc., Assistant lecturer, college of Dentistry, Al-Mustansiriya University. \\ Haitham Dakhil Mohsen \\ B.D.S., M.Sc., Assistant lecturer, College of Dentistry, Al-Mustansiriya University. \\ Sundus Hussein Naser \\ B.D.S., M.Sc., Assistant lecturer, College of Dentistry, Al-Mustansiriya University
}

\begin{abstract}
Aim: The purpose of this study was to determine the number of canals treated in the mesio-buccal roots of the maxillary first molars in Iraqi population.

Materials and Methods: The examiners verified the number of canals in the teeth using different methods including: magnifying lens, bubble test and radio graphical method.

Results: In total, 200 maxillary first molars of which 148 teeth (74\%) met the criterion of having two canals detected and treated in the mesio-buccal root of the maxillary first molars.

Discussion and conclusion: The fact that almost more than half the mesio-buccal roots of the maxillary first molars bear two canals is enough reason to assume always that two canals exist until careful examination proves otherwise. According to this study there was no difference in the prevalence of $2^{\text {nd }} \mathrm{MB}$ canal in maxillary $1^{\text {st }}$ molar between males and females.
\end{abstract}

KEY WORDS : Second cananl, Mazillary first molars,mesial root

CITE THIS ARTCLE

Mohammed A,Mohsen H, Naser S. Detection of Second Canals in Mesial Root of Maxillary First Molars Using Different Evaluation Methods (In Vitro Study) . Iraqi Dent. J. 2016; 38(1):39-42. http://www.iraqidentaljournal.com

\section{INTRODUCTION}

The aim of endodontic treatment is the chemomechanical cleansing of the root canal and its hermetic obturation with an inert material ${ }^{(1)}$. It is generally accepted that the major cause of the failure of root canal therapy is the clinician's inability to recognize and adequately treat all the canals of the root canal system ${ }^{(1-3)}$. A clear understanding of human root canal anatomy is a prerequisite for performing conventional endodontic procedures. The anatomic complexities of the root canal have been highlighted in the literature, and the need for clinicians to understand probable aberrations has been emphasized $(4,5)$.

The morphology of canal systems in maxillary molars fig. 1 has been evaluated in many studies. In particular, the complexity of the mesiobuccal (MB) roots of the first and second maxillary molars, which was first noted by Hess and Zurcher ${ }^{(6)}$, later became the focus of more detailed and repeated investigations after the publication of a study by Weine et al.(2) .

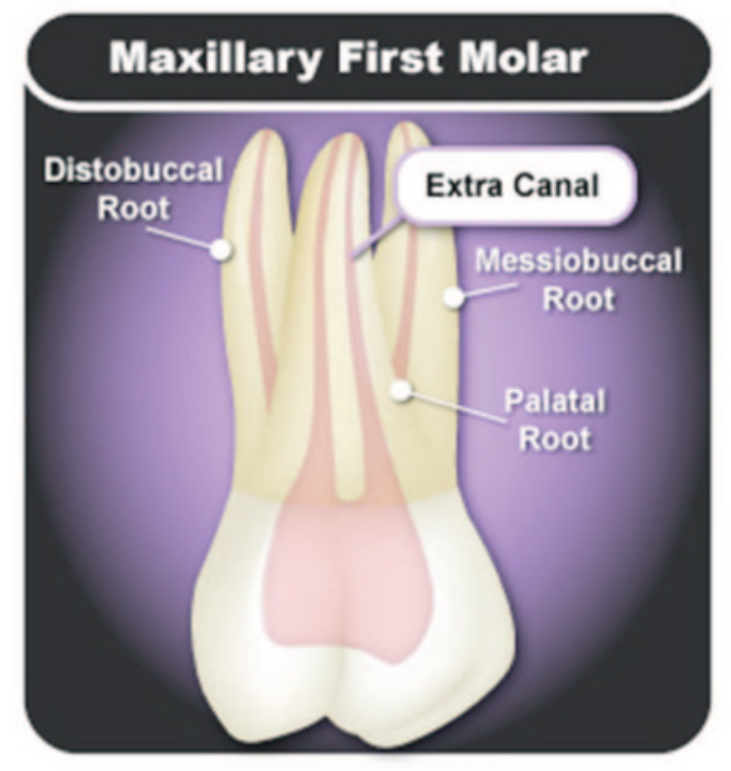

Fig.1 Morphology of maxillary $1^{\text {st }}$ molar

These studies demonstrated that most maxillary first molars and a considerable percent of second molars have an additional MB root canal (MB2), which is also referred to as a mesiolingual canal or a mesiopalatal canal ${ }^{(7)}$. Since these studies, several more studies of the root canal anatomy of maxillary 
molars have shown that the frequency of MB2s ranges from $10 \%$ to $95 \% \quad(1,3-5,8,9)$.

The prevalence of MB2s varies with the method used in the study i.e., the sectioning of extracted teeth, the injection of dye, the study of radiographs, the surgical techniques used, the use of light microscopy or scanning microscopy, microcomputed tomography, and whether the clinical study is retrospective or prospective $^{(4,10,8)}$.

A study by Pomeranz and Fishelberg ${ }^{(3)}$ revealed a large discrepancy between clinical and laboratory results with regard to the incidence of MB2s. Although in vitro studies have also demonstrated that the incidence of MB2s is high, there is no consensus on the number of MB2s that are amenable to endodontic treatment $(12,16)$.

To categorize the root canal system in each root, Weine described four different configurations: type I (a single canal from the pulp chamber to the apex), type II ( 2 separate canals that leave the chamber but merge short of the apex to form a single canal), type III ( 2 separate canals that leave the chamber and exit the root in separate foramina), and type IV (1 canal that leaves the chamber but divides short of the apex into 2 separate and distinct canals with separate foramina). ${ }^{(2)}$ Fig. 2

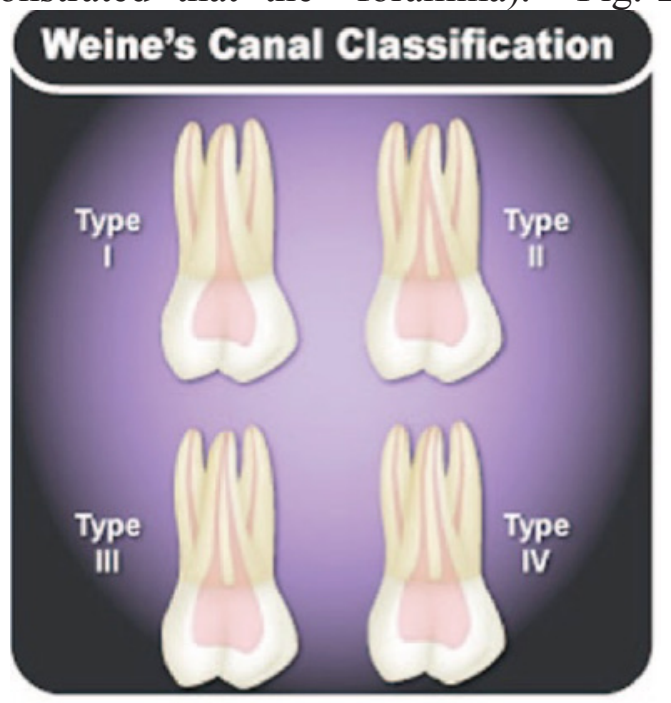

Fig .2 Weine canal classifications

Many authors have discussed different methods of locating extra canals like:

1.Additional off-angle radiographs (at least three radiographs at varying horizontal angles).

2.Use of computed tomography.

3.Use of magnification (loupes and dental operating microscopes).

4.Examining dentinal map minutely and useing DG 16 to explore the floorof the pulp chamber.

5.Looking for hemorrhagic spots (indicate the presence of extra canals).

6.Performing Champaign bubble test by flooding the pulp chamber with warm solution of $5 \%$ sodium hypochlorite, to visualize bubbles emanating from organic tissue indicating presence of canals.

7.Staining the pulp chamber with dye (e.g., $1 \%$ methylene blue).

8.Use of ultrasonic tips, special round burs, and thin tapering finishing burs to remove a small amount of tooth structure or calcification and trough the line angles of the pulp chamber will help.

9.Modifying the conventional outline form to include the extra canals.

10.Ensuring adequate straight-line access to improve visibility $^{(8,9,10)}$.

\section{MATERIALS AND METHODS}

A total of 200 extracted permanent maxillary first molar teeth of Iraqi patients (100 male and100 female) were collected from different health centers and private clinics.

After extraction, all teeth were sectioned at coronal orifice using A diamond disc mounted on straight hand-piece and under water coolant and then stored in $0.1 \%$ thymol solution at room temperature. The anatomic dark lines in the floor of the pulp chamber were examined with a DG16 endodontic explorer (Hu Freiday, Chicago, IL, USA) to identify the root canal orifice.

After locating the orifices, the teeth were placed in $0.5 \%$ sodium hypochlorite solution for 48 hours to dissolve debris and pulp remnants. All the specimens were then thoroughly washed in running water for 4 hours to clean the root canals of any debris.

Patency of each canal was established by passing a k-type file through the apical foramen and canal orifices, for calcified canal initial negotiation wad done using small stainless steel hand files, they are stiffer than equivalent size Nickel Titanium and can be 
pushed through obstacles more easily. At first starting using loupes and magnifying glasses (x2.0 to x6.0) up to the operating microscope $(x 4.0$ to $\times 30.0)$ that aid in detection of number of root canals in mesiobuccal root, A Champaign bubble test was then carried out by flooding the pulp chamber with warm solution of $5 \%$ sodium hypochlorite, one could visualize bubbles emanating from organic tissue indicating presence of canals. We finally verified the number of canals radiographically using multiple straight and angled radiographs allows visualization of the presence of extra canals.

\section{RESULTS}

We collected a total of 200 maxillary first molars over 6 month's period. The results of the study showed that using magnifying glasses $42 \%$ of $2^{\text {nd }} \mathrm{MB}$ canals could be detected while $74 \%$ of canals could be detected using bubble tests and $59 \%$ of canals detected using radiographs (Table 1).

According to the results of the present study, there was no difference in the prevalence of $2^{\text {nd }} \mathrm{MB}$ canal in maxillary $1^{\text {st }}$ molar between male and females.

Table 1 Percentage and number of root canals and apical foramina in the MB root of maxillary first molar using different method.

\begin{tabular}{|c|c|c|c|c|c|}
\hline Method & No. of teeth & Type I\% & Type II\% & Type III\% & Calcified canal\% \\
\hline magnifying glasses & 200 & 58 & 19 & 23 & 0 \\
\hline bubble test & 200 & 26 & 36 & 22 & 16 \\
\hline X-ray & 200 & 41 & 22 & 19 & 18 \\
\hline
\end{tabular}

MB, mesio-buccal.

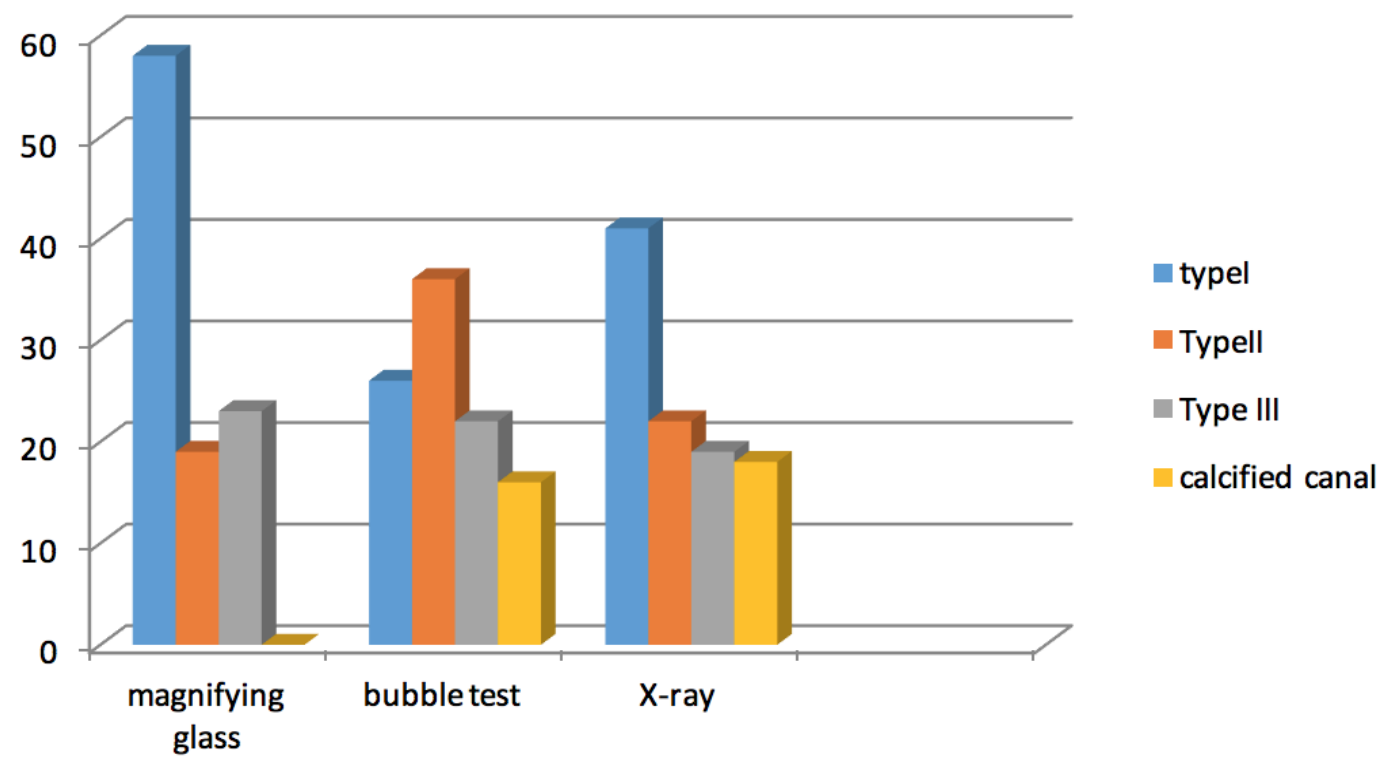

Fig.3 Bar chart for percentage and number of root canals and apicasl foramina in the MB root of maxillary first molar using different methods.

Table2 Association of gender with $2^{\text {nd }} \mathrm{MB}$ canal in maxillary $1^{\text {st }}$ molar teeth

Gender
Male
Female
Total

\section{DISCUSSION}

The goal of this study was to report the percentage of the MB roots of maxillary first molars that contained at least two root canals. The results of this study showed a high prevalence of the MB2 canal in the MB root of maxillary first molars in Iraqi population.

This finding is in agreement with previous

\begin{tabular}{|c|}
\hline No. of teeth \\
\hline 100 \\
100 \\
200
\end{tabular}

$1=$ presence of $2^{\text {nd }} \mathrm{MB}$

studies $(11,12,16)$. An examination of the floor of the pulp chamber offers clues to the type of canal configuration present. When there is only one canal, it is usually located rather easily in the center of the access preparation. If only one orifice is found and it is not in the center of the tooth, it is probable that another canal is present and the operator should search for it on the opposite side ${ }^{(13)}$. 
It is generally accepted that the maxillary first molar has three canals with an MB2 canal seen in $56.8 \%-80.9 \%$ of the cases similar findings were recorded in this study. The closer the orifices are to each other, the greater are the chances that the two canals join at some point within the body of the $\operatorname{root}^{(14}$, ${ }^{15)}$. In this study magnifying lenses were used during the exploration of canal orifices, since magnification has been found to increase the detection rate of MB2 canals from $17.2 \%$ with the naked eye, to $62.5 \%$ with loupes and $71.1 \%$ using the surgical operating microscope ${ }^{(17)}$

The use of radiograph to study the canal morphology might appear to have certain limitations, since it's a two dimensional image of a three dimensional object. However Weine ${ }^{(20)}$ suggests that for the maxillary first molar, angulation from distal to mesial provides a profile type of view of the mesiobuccal root and greater possibility of detection of MB2 canal. In the present study more than $15 \%$ of the teeth presented with different amount of pulp chamber and/or calcified canals.

For easier access to canal openings a bubble test with sodium hypochlorite in the pulp chamber and chelating agents (EDTA) was occasionally used for removing the smear layer and softening calcifications. Ibarrola et al. ${ }^{(19)}$ suggested the use of chelating agents and ultrasonic instrumentation to remove debris and anatomical irrigularities that interfere with negotiation of the MB and ML canals ${ }^{(18)}$.

In this study different methods used for detection of 2nd canal in maxillary 1st molar but the bubble test showed the best results because it was found that When $\mathrm{NaOCl}$ is flooded into the access cavity, it dissociates into $\mathrm{Na}+$ and $\mathrm{Cl}$ - ions and liberates free oxygen. A positive bubble reaction signifies that $\mathrm{NaOCl}$ is reacting with residual tissue within the instrumented or the missed canal or with the residual chelator present within the prepared canal.

There was no difference in the prevalence of $2^{\text {nd }}$ MB canal in maxillary $1^{\text {st }}$ molar between male and females in the present study and this was in agreement with Saad Al-Nazhan ${ }^{(12)}$.

\section{CONCLUSION}

A thorough knowledge of the root canal anatomy, careful interpretation of the radiographs, proper modification of the conventional access cavity were the essentials for recognition and adequate treatment of these anatomical variations. Every tooth has to be treated as an exception and the pulp chamber explored for an additional canal.

\section{REFERENCES}

1. Vertucci FJ. Root canal anatomy of the human permanent teeth. Oral Medicine Oral Pathology Oral Radiology 1984; 58: 589-99.

2. Weine FS, Healey HJ, Gerstein H, Evanson L. Canal configuration in the mesiobuccal root of the maxillary first molar and its endodontic significance. Triple O1969; 28: 419-25.

3. Pomeranz HH, Fishelberg G. The secondary mesiobuccal canal of maxillary molars. J Am Dent Assoc 1974; 88: 11924.

4. Kulild JC, Peters DD. Incidence and configuration of canal systems in the mesiobuccal root of maxillary first and second molars. J Endod 1990; 16: 311-17.

5. Pineda F, Kuttler Y. Mesiodistal and buccal lingual roentgenographic investigation of 7,275 root canals. Triple O 1972; 33: 101-10.

6. Hess W, Zurcher E. The Anatomy of the Root Canals of the Teeth of the Permanent and Deciduous Dentitions. New York: William Wood \& Co., 1925; 1-39.

7. Alavi AM, Opasanon A, Ng YL, Gulabivala K. Root and canal morphology of Thai maxillary molars. Int Endod J 2002; 35: 478-85.

8. Johal S. Unusual maxillary first molar with 2 palatal canals within a single root: a case report. J Can Dent Assoc 2001;67:211-4

9. Smadi L, Khraisat A. Detection of a second mesiobuccal canal in the mesiobuccal roots of maxillary first molar teeth. Triple O 2007;103:77-81.

10. Holderrieth S, Gernhardt CR. Maxillary molars with morphologic variations of the palatal root canals: a report of four cases. J Endod 2009;35:1060-5.

11. Bestoon M. Faraj. The Incidence of Two Root Canals in the Mesial Root of the Extracted Permanent Maxillary First Molars Among a Sulaimani-Iraq Population. Tikrit J for Dent Sciences 2012;2:106-109.

12. Saad Al-Nazhan. The prevalence of two canals in mesial root of endodontically treated maxillary first molars among a Saudi Arabian sub-population. Saudi Dent J. 2005; 17: $15-21$.

13. Acosta V. and Trugeda B. Anatomy of the pulp chamber floor of the permanent maxillary first molar. J Endod .1978; 4: 214-219.

14. Cleghorn B. Christie H. and Dong C. Root and root canal morphology of the human permanent maxillary first molar: a literature review. J Endod.2006; 32; 9: 813-820.

15. Imura N. Hata G. Toda T. Otani S. and Fagundes M. Two canals in mesiobuccal roots of maxillary molars. Int End J.1998; 31; 6: 410-414.

16. F. Wasti, A. C. Shearer,N. H. F. Wilson. Root canal systems of the mandibular and maxillary first permanent molar teeth of South Asian Pakistanis.Int End J. 2001;34:263-266.

17. Buchrley L. Barrows M. BeGole E.and Wenckus C. Effect of magnification on locating the MB2 canal in maxillary molars. J Endod .2002; 28: 324-327.

18. Emel O. Mete Ü.and Ersin Ö. Canal Configuration of the Mesiobuccal Root of Maxillary Molars. Hacettepe Diş Hekimliği Fakültesi Dergisi. 2008; 32: 2-9.

19. Ibarrola J. Knowles K. Ludlow M.and Mickinley B.Factors affecting the negotiability of second mesiobuccal canals in maxillary molars. J Endod .1997; 23: 236-238.

20. Weine FS. Endodontic Therapy. 5th ed. St Louis, Mo: Mosby; 1996. 